PROCEEDINGS OF THE

AMERICAN MATHEMATICAL SOCIETY

Volume 134, Number 2, Pages 501-507

S 0002-9939(05)08021-4

Article electronically published on July 8, 2005

\title{
FIXED POINT THEOREMS UNDER THE INTERIOR CONDITION
}

\author{
ANTONIO JIMÉNEZ-MELADO AND CLAUDIO H. MORALES
}

(Communicated by Joseph A. Ball)

\begin{abstract}
We show a fixed point theorem for condensing mappings under a new condition of the Leray-Schauder type. We call it the Interior Condition. We also discuss examples that demonstrate the independence of these two conditions.
\end{abstract}

\section{IntRoduction}

Let $X$ be a real Banach space, $G$ an open subset of $X$ with $0 \in G$ and $T$ a mapping defined on the closure $\bar{G}$ of $G$ and taking values in $X$. It is well known that if $G$ is bounded and if $T$ is a condensing mapping, then the Leray-Schauder boundary condition

$$
T(x) \neq \lambda x \text { for } x \in \partial G \text { and } \lambda>1
$$

( $\partial G$ denotes the boundary of $G$ ) is sufficient to guarantee existence of a fixed point for $T$. Indeed, this result is due to Petryshyn Pe 71] who observed that it follows easily from Nussbaum's degree theory $\mathrm{Nu}$. For an elegant non-degree-theoretic proof see $\underline{\mathrm{Sch}}$.

In this paper, we introduce a new condition which resembles the Leray-Schauder condition mentioned above. However, in this case, it holds for the interior points of $G$, which lie near to the boundary of $G$. Specifically, this interior condition may be formulated as follows.

We say that a mapping $T: \bar{G} \rightarrow X$ satisfies the Interior Condition if there exists $\delta>0$ such that

$$
T(x) \neq \lambda x \text { for } x \in G^{\star}, \lambda>1 \text { and } T(x) \notin \bar{G},
$$

where $G^{\star}=\{x \in G$ : $\operatorname{dist}(x, \partial G)<\delta\}$.

Following $[\mathrm{Ku}$, we define the set-measure of noncompactness of a bounded subset $A$ of $X$ as

$\gamma[A]=\inf \{d>0: A$ can be covered by finitely many sets of diameter $\leq d\}$.

Received by the editors June 23, 2004 and, in revised form, September 27, 2004.

2000 Mathematics Subject Classification. Primary 47H10, 47H09.

Key words and phrases. Condensing mappings, nonexpansive mappings, Leray-Schauder condition, interior condition, Banach spaces.

This research was partially supported by a Grant from Ministerio de Educación y Ciencia, Spain (MTN 2004-00078), and from La Junta de Andalucía (FQM210).

(C)2005 American Mathematical Society 
In what follows, some well-known properties of $\gamma$ will be needed, and these are: $\gamma[A] \leq \gamma[B]$ whenever $A \subset B, \gamma[A \cup B]=\max \{\gamma[A], \gamma[B]\}, \gamma[\overline{c o}(A)]=\gamma[A]$, $\gamma[\lambda A]=|\lambda| \gamma[A]$ and $\gamma[A]=0$ if and only if $\bar{A}$ is compact. A mapping $T: G \rightarrow X$ is called a condensing mapping (see [Sa] ) if it is continuous and $\gamma[T(A)]<\gamma[A]$ for all bounded subsets $A$ of $G$ whose $\gamma[A]>0$. However, if $k>0$, we say that $T: G \rightarrow X$ is $k$-set-contractive if $T$ is continuous and $\gamma[T(A)] \leq k \gamma[A]$ for each bounded set $A \subset G$. On the other hand, if $k=1, T$ is called a 1-set-contraction. It follows that every condensing mapping, as well as every nonexpansive mapping, is 1-set-contractive (recall that $T$ is nonexpansive if $\|T(x)-T(y)\| \leq\|x-y\|$ for all $x, y \in G)$.

In section 2 , we observe that a fixed point theorem similar to the one obtained by Petryshyn does not necessarily hold true under this new Interior Condition. In fact, it fails whenever $G$ is an arbitrary bounded open set, even for the case that $G$ is a star-shaped set. Then we introduce a type of domain, which appears to be the best in this context, for the purpose of obtaining fixed point results. To end the section we prove that this class of domains remains between the star-shaped sets and the convex sets.

In section 3, we state and prove our main result for condensing mappings satisfying the condition (I-C) and, following standard arguments, we derive a result for nonexpansive mappings defined on uniformly convex spaces.

In section 4, we compare the conditions (L-S) and (I-C). We exhibit some examples which show that, in general, none of these conditions implies the other, even for compact operators. Despite this, we show that for nonexpansive mappings in Hilbert space, condition (I-C) implies condition (L-S).

\section{THE INTERIOR CONDITION}

We begin this section with an example of a fixed point free compact operator which satisfies the Interior Condition on a star-shaped set with respect to the origin.

Example 1. Consider the euclidean space $\mathbb{R}^{2}$ and let $G$ be a subset of $\mathbb{R}^{2}$ obtained by removing from the open unit disk the closed segment of vertices $\left(0, \frac{1}{2}\right)$ and $(0,1)$. Define $T: \bar{G} \rightarrow \mathbb{R}^{2}$ as $T(a, b)=(a, b)+\left(0, \frac{1}{2}\right)$. Then $G$ is a bounded open starshaped set with respect to the origin and $T$ satisfies the Interior Condition, but $T$ has no fixed point in $\bar{G}$.

A routine computation would allow the reader to verify the details of the above example. We remark that the crucial fact used to obtain this somewhat disappointing example is that the boundary of $G$ contains the segment $\left[\left(0, \frac{1}{2}\right),(0,1)\right]$. Consequently, there is a need for a more restricted domain for the operator $T$. We introduce a class of star-shaped sets whose boundary does not contain segments.

Definition 1. Let $X$ be a Banach space and let $G$ be a bounded open subset of $X$ with $0 \in G$. We say that $G$ is strictly star-shaped with respect to the origin if for each $x \in \partial G,\{t x: t>0\} \cap \partial G=\{x\}$.

From now on, we will assume without lost of generality, that the star-shaped assumption will always be considered with respect to the origin, unless the contrary is mentioned.

Proposition 1. Let $X$ be a Banach space and let $G$ be a bounded open subset of $X$ with $0 \in G$. 
(a) If $G$ is convex, then it is strictly star-shaped.

(b) If $G$ is strictly star-shaped, then it is star-shaped.

Proof. To prove (a) suppose that $G$ is convex but not strictly star-shaped. Then there exist $x \in \partial G$ and $0<t_{2}<t_{1}$ such that $t_{i} x \in \partial G, i=1,2$. Since $G$ is open and $0 \in G$, there exists $r>0$ such that $B(0 ; r) \subset G$ and we may choose $u \in G$ with $\left\|u-t_{1} x\right\|<r\left(t_{1}-t_{2}\right) t_{2}^{-1}$. Then the point $v=t_{2}\left(t_{1}-t_{2}\right)^{-1}\left(t_{1} x-u\right)$ belongs to $G$, from which we conclude that $t_{2} x \in G$ because $G$ is convex and $t_{2} x=\left(t_{1}-t_{2}\right) t_{1}^{-1} v+t_{2} t_{1}^{-1} u$. This contradicts that $t_{2} x \in \partial G$, since $G$ is open.

Now, we prove part (b). Suppose that $G$ is not star-shaped. Then there exists $x \in G$ and $0<\rho<1$ such that $\rho x \notin G$. Define $t_{0}$ and $t_{1}$ by $t_{0}=\sup \{t>0$ : $s x \in G$ for all $s \in[0, t]\}$ and $t_{1}=\sup \{t>0: t x \in G\}$. Then $t_{0} x, t_{1} x \in \partial G$ and $t_{0} x \neq t_{1} x$, contradicting that $G$ is strictly star-shaped.

\section{A FIXED POINT THEOREM}

An important step in the proof of our main theorem is the well-known fixed point theorem of Petryshyn Pe 71, mentioned earlier, which for the sake of completeness we state as a lemma.

Lemma 1 (Proposition 1 of Pe 71]). Let $X$ be a real Banach space, $G$ a bounded open subset of $X$ with $0 \in G$ and $T: \bar{G} \rightarrow X$ a condensing mapping satisfying the condition (L-S). Then $T$ has a fixed point in $\bar{G}$.

Theorem 1. Let $X$ be a real Banach space and let $G$ be a bounded open and strictly star-shaped subset of $X$ with $0 \in G$. If $T: \bar{G} \rightarrow X$ is a condensing mapping satisfying the condition (I-C), then $T$ has a fixed point in $\bar{G}$.

Proof. Let $M=\sup \{\|z\|: z \in \partial G\}$. Since $T$ satisfies the condition (I-C), there exists $\delta \in(0, M)$ such that $T(x) \neq \lambda x$ whenever $\lambda>1, T(x) \notin \bar{G}$ and $x \in G^{\star}=$ $\{x \in G: \operatorname{dist}(x, \partial G)<\delta\}$. Let $t \in\left(1-\frac{\delta}{M}, 1\right)$. Then by the assumptions on $G$, the set $G_{t}=\{t x: x \in G\}$ is an open subset of $G$, and $\overline{G_{t}}=t \bar{G}$. Additionally, $t \bar{G} \subset G$. Otherwise, there would exist $x \in \bar{G}$ with $t x \notin G$, which would imply that $x, t x \in \partial G$. However, this would contradict the fact that $G$ is strictly star-shaped since $t<1$.

Therefore, we may assume that there exists $x_{t} \in \partial G_{t}$ such that

$$
T\left(x_{t}\right)=\lambda_{t} x_{t} \quad \text { for some } \lambda_{t}>1 \text {; }
$$

otherwise, due to Lemma 1, T would have a fixed point in $\bar{G}$ and hence the proof would be complete. Now, using the fact that $G$ is strictly star-shaped and that $x_{t} \in \partial G_{t} \subset G$, we obtain the existence of a unique $z_{t} \in \partial G$ such that $x_{t}=t z_{t}$. Then we have that

$$
d\left(x_{t}, \partial G\right) \leq\left\|x_{t}-z_{t}\right\|=(1-t)\left\|z_{t}\right\| \leq(1-t) M<\delta,
$$

and consequently, by the condition (I-C), $\lambda_{t} x_{t} \in \bar{G}$. Using again that $G$ is strictly star-shaped, we conclude that $\lambda_{t} x_{t} \in \operatorname{seg}\left[x_{t}, z_{t}\right]$, which implies that

$$
\left\|x_{t}\right\|<\left\|\lambda_{t} x_{t}\right\| \leq\left\|z_{t}\right\| \text {. }
$$

Therefore, $t\left\|z_{t}\right\|<t \lambda_{t}\left\|z_{t}\right\| \leq\left\|z_{t}\right\|$. Since the $z_{t}$ with $t \in(0,1)$ are bounded away from the origin, $\lambda_{t} \rightarrow 1^{+}$as $t \rightarrow 1^{-}$. Define the subset $E$ as

$$
E=\left\{x_{t} \in G: x_{t} \text { satisfies }(1), 1-\delta / M<t<1\right\} .
$$


Then $E \subset \overline{\mathrm{co}}(T(E) \cup\{0\})$. However, since $T$ is condensing, $E$ is precompact, and hence there exists a convergent sequence $\left\{x_{n}\right\}$ in $\bar{G}$, say, $x_{n} \rightarrow x$, and a sequence $\left\{\lambda_{n}\right\}$ in $\mathbb{R}$ such that

$$
T\left(x_{n}\right)=\lambda_{n} x_{n} \quad \text { with } \lambda_{n} \rightarrow 1^{+} .
$$

Therefore, $x$ is a fixed point of $T$, which completes the proof.

In the next corollary we first recall that our result includes the convex case and then, following the pattern of Petryshyn $\mathrm{Pe} 73$, we give a fixed point theorem for nonexpansive mappings satisfying the Interior Condition in the setting of uniformly convex spaces. The essential key will be a result of Browder $\mathrm{Br}$ which asserts that if $T: M \rightarrow X$ is a nonexpansive mapping defined on a closed, bounded and convex subset $M$ of a uniformly convex space $X$, then $I-T$ is demiclosed. This means that if $\left(x_{n}\right)$ is a sequence in $M$ which converges weakly to $x$ and $x_{n}-T\left(x_{n}\right) \rightarrow y$, then $x-T(x)=y$.

Corollary 1. Let $X$ be a real Banach space, $G$ a bounded and open subset of $X$, with $0 \in G$, and $T: \bar{G} \rightarrow X$ a mapping satisfying the condition (I-C). Then we have:

(a) If $G$ is convex and $T$ is condensing, then $T$ has a fixed point in $\bar{G}$.

(b) If $G$ is strictly star-shaped and $T$ is 1-set-contractive, then there exists a sequence $\left\{x_{n}\right\}$ in $\bar{G}$ such that $x_{n}-T\left(x_{n}\right) \rightarrow 0$.

(c) If $G$ is convex, $T$ is nonexpansive and $X$ is uniformly convex, then $T$ has a fixed point in $\bar{G}$.

Proof. Part (a) follows from Proposition 1 and Theorem 1. To prove part (b) define, for any positive integer $n, T_{n}: \bar{G} \rightarrow X$ as $T_{n}(x)=\left(1-\frac{1}{n}\right) T(x)$. It is clear that $T_{n}$ is condensing since, in fact, it is $k$-set-contractive with $k=\left(1-\frac{1}{n}\right)$. Also $T_{n}$ satisfies the condition (I-C) since $T$ itself satisfies the same condition and $G$ is star-shaped. Then, by Theorem 1 , there exists $x_{n} \in \bar{G}$ such that $x_{n}=T_{n}\left(x_{n}\right)=\left(1-\frac{1}{n}\right) T\left(x_{n}\right)$. Hence, $x_{n}-T\left(x_{n}\right) \rightarrow 0$, since $\bar{G}$ is bounded.

To prove part (c) we follow standard arguments. A result of Browder $\mathrm{Br}$ guarantees that $I-T$ is demiclosed because $\bar{G}$ is closed, bounded and convex, $T$ is nonexpansive and $X$ is uniformly convex. On the other hand, $T$ is 1 -set-contractive because it is nonexpansive and hence there exists a sequence $\left\{x_{n}\right\}$ in $\bar{G}$ such that $x_{n}-T\left(x_{n}\right) \rightarrow 0$. Since $X$ is reflexive, $\left\{x_{n}\right\}$ has a weakly convergent subsequence, and thus the demiclosedness of $I-T$ implies that $T$ has a fixed point.

\section{Comparison between (L-S) and (I-C)}

To start this section we give some examples which show that none of the geometric conditions studied in this paper implies the other.

Example 2. Let $X$ be $\mathbb{R}^{2}$ with its usual euclidean norm, $\|\cdot\|$, and let $K$ be the closed unit ball, $K=\left\{x \in \mathbb{R}^{2}:\|x\| \leq 1\right\}$. Define $T: K \rightarrow X$ as $T(x)=2 A(\|x\|) x$, where for $t \in \mathbb{R}, A(t)$ is the matrix

$$
A(t)=\left(\begin{array}{cc}
\cos (2 \pi t) & -\sin (2 \pi t) \\
\sin (2 \pi t) & \cos (2 \pi t)
\end{array}\right)
$$

Then $T$ satisfies the Interior Condition but not the Leray-Schauder Condition. 
$T$ does not satisfy the Leray-Schauder Condition because for any $x$ in the unit sphere we have $T(x)=2 x$. Let us see that $T$ satisfies the Interior Condition. Indeed, we shall prove that $T(x) \neq \lambda x$, whenever $\lambda>0,0<\|x\|<1$ and $\|T(x)\|>1$ : suppose on the contrary that $T(x)=\lambda x$, for some $\lambda>0,0<\|x\|<1$ and $\|T(x)\|>1$. Since $x \neq 0$ and $\|T(x)\|=2\|x\|$, we have that $\lambda=2$ and then $A(\|x\|) x=x$, with $x \neq 0$. Hence, the matrix

$$
\left(\begin{array}{cc}
\cos (2 \pi\|x\|)-1 & -\sin (2 \pi\|x\|) \\
\sin (2 \pi\|x\|) & \cos (2 \pi\|x\|)-1
\end{array}\right)
$$

has null determinant, which is impossible because $0<\|x\|<1$.

The mapping $T$ in the above example does satisfy the Leray-Schauder condition in any ball $B(0 ; r)$, with $0<r<1$, but it can be used to provide an example without this deficiency.

Example 3. Let $E$ be the usual euclidean space $\left(\mathbb{R}^{2},\|\cdot\|\right)$ and consider the space $X=\ell_{\infty}(E)$ of all sequences $x=\left(x_{n}\right)$ with $x_{n} \in E$ and $\|x\|_{\infty}=\sup _{n>1}\left\|x_{n}\right\|<\infty$. It is well known that $X$ is a Banach space with the norm $\|\cdot\|_{\infty}$.

Let $B$ be the closed unit ball of $X$ and define $H: B \rightarrow X$ as $[H(x)]_{n}=T_{n}\left(x_{n}\right)$, where $T_{1}=T$, as defined in Example 2, and

$$
T_{n}(u)= \begin{cases}\frac{1}{n-1} \frac{u}{\|u\|} & \text { if } \frac{1}{n} \leq\|u\| \leq 1 \\ \frac{1}{2(n-1)} T(n u) & \text { if }\|u\|<\frac{1}{n}\end{cases}
$$

for $n \geq 2$. Then $H$ is a compact operator that satisfies the Interior Condition in $B$ but it does not satisfy the Leray-Schauder condition in any ball $B(0 ; r)$, with $0<r \leq 1$.

To see that $H$ does not satisfy the Leray-Schauder condition in $B(0 ; r)$ for any $r \in(0,1]$, we first observe that for $r=1$ the assertion follows from the above Example 2. Suppose now that $0<r<1$ and select a positive integer $n \geq 2$ with $\frac{1}{n} \leq r<\frac{1}{n-1}$. Consider the element $x \in \partial B(0 ; r)$ defined as $x_{i}=(0,0)$ for $i \neq n$ and $x_{n}=(r, 0)$. Then

$$
[H(x)]_{i}= \begin{cases}(0,0) & \text { if } i \neq n \\ \left(\frac{1}{n-1}, 0\right) & \text { if } i=n\end{cases}
$$

and hence $H(x)=\lambda x$, with $\lambda=\frac{1}{r(n-1)}>1$.

Next, we verify that $H$ satisfies the Interior Condition in $B$. Suppose that there exists $x \in B$ with $0<\|x\|_{\infty}<1$ such that $H(x)=\lambda x$, for some $\lambda>1$ and $\|H(x)\|_{\infty}>1$. Since $\left\|T_{i}\left(x_{i}\right)\right\| \leq 1$ for all $i \geq 2$, we should have $\left\|T_{1}\left(x_{1}\right)\right\|>1$ with $T_{1}\left(x_{1}\right)=\lambda x_{1}$. Observe that we also have that $0<\left\|x_{1}\right\|<1$, which enters in contradiction with what was proved for the mapping $T=T_{1}$ in Example 2 above.

The compactness of the operator $H$ can be argued as follows: first of all, its continuity is a consequence of the fact that each $T_{i}$ is continuous and the fact that $\operatorname{diam} T_{n}(K) \rightarrow 0$. Secondly, by observing that $H(B) \subset T_{1}(K) \times T_{2}(K) \times \cdots$, where $K$ is the closed unit ball in $E$ and $\operatorname{diam}_{n}(K) \rightarrow 0$, we may derive that $H(B)$ is relatively compact.

Example 4. Consider the mapping $T:[-1,1] \rightarrow \mathbb{R}$ given by $T(x)=2-x^{2}$. Then $T$ satisfies the Leray-Schauder Condition but not the Interior Condition. 
Clearly $T$ satisfies the Leray-Schauder condition since $|T(x)|=|x|$ if $|x|=1$. On the other hand, $T$ does not satisfy the Interior Condition. This follows from the fact that for any $x$ with $0<|x|<1$ we have $T(x)>1$.

Remark 1. If $T:[-1,1] \rightarrow \mathbb{R}$ is continuous, then $T$ satisfies the Leray-Schauder condition whenever $T$ satisfies the Interior Condition. The converse is not true, as Example 4 shows. However, for a Hilbert space the former implication holds when $T$ is nonexpansive, as is shown in the following theorem.

Theorem 2. If $X$ is a Hilbert space, $B$ is the closed unit ball of $X$ and $T: B \rightarrow X$ is nonexpansive, then $T$ satisfies the Leray-Schauder condition whenever $T$ satisfies the Interior Condition.

Proof. Suppose that $T$ does not satisfy the Leray-Schauder condition. Then there exists a point $x \in \partial B(0 ; 1)$ and a real number $\lambda>1$ such that $T(x)=\lambda x$. Select an arbitrary real number $\delta$ such that $0<\delta \leq \min \left\{\frac{1}{2}, \frac{1}{3}\|T(x)-x\|\right\}$, and define $K=\partial B\left(0 ; 1-\delta^{2}\right) \cap \bar{B}(x ; \delta)$. Then $K \neq \emptyset$ and $\bar{B}(T(x) ; \delta) \cap B(0 ; 1)=\emptyset$.

Consider the radial projection $R: X \rightarrow \bar{B}(0 ; 1)$ given by

$$
R(u)=\left\{\begin{array}{cc}
\frac{u}{\|u\|} & \text { if }\|u\| \geq 1 \\
u & \text { if }\|u\| \leq 1
\end{array}\right.
$$

and define $h: K \rightarrow X$ by $h(u)=\left(1-\delta^{2}\right) R(T(u))$. We claim that $h(K) \subset K$. To see this, we first observe that since $T$ is nonexpansive, then $T(K) \subset \bar{B}(T(x) ; \delta)$ and consequently $h(K) \subset \partial B\left(0 ; 1-\delta^{2}\right)$. It just remains to show that $h(K) \subset \bar{B}(x ; \delta)$. To see this, let $r=1-\delta^{2}$. Then

$$
\begin{aligned}
\|h(u)-x\|^{2} & =r^{2}+1-2 r \operatorname{Re}\langle R(T(u)), R(T(x))\rangle \\
& =r^{2}+1+r\left(\|R(T(u))-R(T(x))\|^{2}-\|R(T(u))\|^{2}-\|R(T(x))\|^{2}\right) \\
& \leq r^{2}+1+r\left(\delta^{2}-2\right)=\delta^{2} .
\end{aligned}
$$

Therefore $h(K) \subset K$. Since $h$ is also a contraction, it follows that $h$ has a fixed point $x_{0} \in K$. This means

$$
x_{0}=\left(1-\delta^{2}\right) \frac{T\left(x_{0}\right)}{\left\|T\left(x_{0}\right)\right\|},
$$

which implies that $\left\|x_{0}\right\|=1-\delta^{2}$ and $T\left(x_{0}\right)=\rho x_{0}$, with $\rho=\frac{\left\|T\left(x_{0}\right)\right\|}{1-\delta^{2}}>1$ and $T\left(x_{0}\right) \notin B$, which contradicts the Interior Condition.

\section{REFERENCES}

[Br] F. E. BROWDER, Semicontractive and semiaccretive nonlinear mappings in Banach spaces. Bull. Amer. Math. Soc. 74 (1968), 660-665. MR0230179 (37:5742)

[Ku] K. Kuratowski, Sur les espaces complets, Fund. Math. 15 (1930), 301-309.

$[\mathrm{Nu}] \quad$ R. D. Nussbaum, The fixed point index and fixed point theorems for $k$-set-contractions, Univ. of Chicago, Ph. D. thesis, 1969.

[Pe 71] W. V. Petryshyn, Structure of the fixed points sets of $k$-set-contractions, Arch. Ration. Mech. Anal. 40 (1970/71), 312-328. MR0273480(42:8358)

[Pe 73] W. V. Petryshyn, Fixed point theorems for various classes of 1-set-contractive and 1-ball-contractive mappings in Banach spaces, Trans. Amer. Math. Soc. 182 (1973), 323352. MR0328688(48:7030) 
[Sa] B. N. SadovskiI, On a fixed point principle, Funktsional. Anal. i Prilozhen. 1(2) (1967), 74-76. MR0211302 (35:2184)

[Sch] R. SchönEBERG, Leray-Schauder principles for condensing multivalued mappings in topological linear spaces, Proc. Amer. Math. Soc. 72 (1978), 268-270. MR0507320|(81b:47075)

Departamento de Análisis Matemático, Facultad de Ciencias, University of Málaga, 29071 MÁlaga, Spain

E-mail address: melado@uma.es

Department of Mathematics, University of Alabama in Huntsville, Huntsville, AlABAMA 35899

E-mail address: morales@math.uah.edu 